\title{
Fontes de autoeficácia em estudantes do ensino superior
}

\author{
Maria Odilia Teixeira ${ }^{1}$ \\ Cátia João Costa \\ Faculdade de Psicologia da Universidade de Lisboa, Lisboa, Portugal
}

\section{Resumo}

Numa perspetiva sociocognitiva, analisam-se fontes de autoeficácia, em variáveis demográficas, académicas, vocacionais e personalidade. A amostra inclui 480 estudantes do $2^{\circ}$ ano dos cursos de psicologia, saúde, desporto, científicos-investigativos e tecnologias, que responderam ao Questionário Ajustamento e Adaptação ao Ensino Superior, Escalas de Desenvolvimento e Bem-Estar, Satisfação na Carreira, Autoeficácia Geral, Exigência e Responsividade Parental. Nos dados da regressão múltipla linear são variáveis explicativas das Autoeficácia: média de acesso ao ensino superior, abertura à experiência (experiências), bem-estar, satisfação na carreira, neuroticismo (estados emocionais), amabilidade, exigência paterna (persuasão, experiência vicária), e idade e género. Discutem-se implicações dos dados nas intervenções educacionais.

Palavras-chave: Autoeficácia, fontes de autoeficácia, aprendizagem

\section{Abstract: Self-efficacy in higher education students}

With a socio-cognitive framework, we analyze sources of General Self-Efficacy, in demographic, academic, vocational and personality variables. The sample includes 480 students of the 2 nd year of psychology, health, sports, scientificresearch and technology courses who answered the Questionnaire Adjustment and Adaptation to Higher Education, Scales of Development and Well-being, Career Satisfaction, General Self-Efficacy, Requirement and Responsiveness Parental. In the multiple linear regression data are explanatory variables of Self-efficacy: average access to higher education, openness to experience (experiences), well-being, career satisfaction, neuroticism (emotional states), kindness, paternal requirement (persuasion, vicarious experience), and age and gender. Implications of data on educational interventions are discussed.

Keywords: Self-efficacy, sources of self-efficacy, learning

\section{Resumen: Fuentes de autoeficacia en estudiantes de enseñanza superior}

En una perspectiva sociocognitiva se analizan fuentes de autoeficacia, en variables demográficas, académicas, vocacionales y personalidad. La muestra incluye 480 estudiantes del $2^{\circ}$ año de los cursos de psicología, salud, deporte, científicos-investigativos y tecnologías, que respondieron al Cuestionario Ajuste y Adaptación a la Enseñanza Superior, Escalas de Desarrollo y Bienestar, Satisfacción en la Carrera, Autoeficacia General, Exigencia y la Responsabilidad parental. En los datos de regresión múltiple lineal son variables explicativas de las Autoeficacia: media de acceso a la enseñanza superior, apertura a la experiencia (experiencias, bienestar, satisfacción en la carrera, neuroticismo (estados emocionales), amabilidad, exigencia paterna (persuasión, experiencia vicaria), y edad y género. Se discuten las implicaciones de los datos en las intervenciones educativas.

Palabras clave: Autoeficacia, fuentes de autoeficacia, aprendizaje

\footnotetext{
${ }^{1}$ Endereço para correspondência: Faculdade de Psicologia. Universidade de Lisboa. Alameda da Universidade, 1649-013, Lisboa, Portugal. E-mail: moteixeira@psicologia.ulisboa.pt
} 
No curso da vida, o ensino superior representa um mundo de oportunidades, em que interagem múltiplos fatores acadêmicos e sociais, que podem facilitar ou impedir florescimento, desenvolvimento pessoal e sucesso académico. Esta etapa escolar exige capacidade de adaptação a diferentes papéis e responsabilidades, que mobilizam recursos internos como autorregulação, autoeficácia, traços de personalidade e competências psicossociais. No domínio das vivências acadêmicas, a literatura mostra o período de adaptação como potenciador de desenvolvimento, bem-estar e individualização mas também de ansiedade e outras formas de sofrimento psicológico (Almeida, Araújo \& Martins, 2016; Ferreira \& Fernandes, 2015). Há evidências da ligação entre estresse e depressão (e.g., Alvan, Belgrave \& Zea, 1996), e entre estabilidade emocional, bem-estar e saúde mental (Almeida, 2017; Silva \& Nascimento, 2014). A questão da adaptação ao ensino superior e dos recursos pessoais associados é central em Psicologia da Educação, pelas repercussões no estudante, na comunidade escolar e familiar e até no próprio país, envolvendo domínios da saúde, aprendizagem e desenvolvimento económico e social.

$\mathrm{Na}$ perspetiva sociocognitiva de Bandura (1977, 1995, 1997), a autoeficácia é uma das dimensões do autoconceito, com papel chave nos processos adaptativos responsáveis pela aprendizagem, motivação, sucesso acadêmico e bem-estar. As crenças de autoeficácia suportam, como mediador cognitivo, a relação das variáveis de natureza pessoal e ambiental. Autoeficácia representa a perceção de capacidades para lidar com situações e usar recursos, especialmente em situações com desafios e obstáculos (Bandura, 1977), como é o caso da adaptação ao ensino superior. Autoeficácia assume-se como força mobilizadora para atingir controlo, volição, persistência e sucesso em alcançar resultados (Bandura, 1997). Autoeficácia é normalmente entendida como contextualizada em tarefas específicas (Luszczynska, Scholz \& Schwarzer, 2005) como, por exemplo, autoeficácia em matemática ou decisão de carreira. No entanto, autoeficácia também pode ser conceptualizada como uma crença mais geral face aos desafios de vida, que se refere a um sentido mais alargado e estável (Luszczynska et al., 2005; Schwarzer \& Jerusalem, 1995). A autoeficácia geral será então a crença na competência pessoal para lidar com um espetro grande de exigências estressantes e desafiantes, enquanto autoeficácia específica está restrita a tarefas particulares. O conceito mais geral é útil em ambientes de transição, quando o foco recai sobre múltiplos comportamentos em simultâneo (Luszczynska, Gibbons, Piko, \& Tekozel, 2004; Luszczynska et al., 2005). Contudo, mesmo nesta aceção mais geral, a perceção de autoeficácia varia conforme as exigências da situação e da predisposição da pessoa para o sucesso e dos estados psicológicos como cansaço, alegria, ansiedade, apatia ou desânimo (Bandura, 1997). A pesquisa tem demonstrado que a autoeficácia geral é um dos fatores fortemente relacionados com sentimentos de bem-estar, satisfação e desenvolvimento pessoal (Almeida \& Teixeira, 2018; Pinto \& Teixeira, 2018; Teixeira \& Costa, 2017). No domínio acadêmico, a investigação tem também demonstrado o efeito das crenças na motivação, sucesso académico e autorregulação (Bandura et al., 2001), e ainda no envolvimento escolar, adaptação e resistência face às dificuldades, especialmente em grupos minoritários (Høigaard, Kovac, Øverby \& Haugen, 2015). No domínio vocacional, o papel da autoeficácia tem sido evidenciado no âmbito dos papéis de vida (Roche, Deskalova \& Brown, 2017; Teixeira, 2008), nos níveis de aspiração (Lopes \& Teixeira, 2012), e em projetos científicos e trajetórias não tradicionais de gênero nas mulheres (Betz \& Hackett, 1981; Pajares, 2002).

A autoeficácia emerge em processos de aprendizagem alicerçados em quatro fontes principais: experiências de mestria, aprendizagem vicariante, persuasão verbal-social e estados físicos e emocionais (Bandura, 1997; Usher \& Pajares, 2009). Estas fontes de autoeficácia atuam em conjunto e influenciam-se reciprocamente ao longo do desenvolvimento (Usher \& Pajares, 2009), sendo a informação selecionada e incorporada nos julgamentos pessoais de capacidade, de acordo com as caraterísticas dos grupos, em idade e ambientes acadêmicos, sociais e culturais (Ahn, Usher, Butz, \& Song, 2016; Chen \& Usher, 2013). Neste âmbito, a escola e a família proporcionam multiplicidade de oportunidades de aprendizagem social e acadêmica, em situações, modelos, expectativas e feedback que oferecem (Ahn, et al, 2016; Lent \& Brown, 2006). A pesquisa mostra a influência do nível socioeconómico e dos fatores culturais da família na adaptação (Batista, 2015; Eurydice/Cedefop, 2015), bem como o efeito do estilo de parentalidade adoptado em termos de educação e socialização. $\mathrm{O}$ estilo da parentalidade é um dos principais agentes de desenvolvimento, ao longo da vida (Hill \& Wang, 2015), nomeadamente na relação entre responsividade, suporte na autonomia e bem-estar (Hutz \& Bardagi, 2006; Pesch, Larson \& Surapaneni, 2016; Teixeira \& Costa, 2017). Nestas relações, são inferidos papéis diferenciados segundo o género dos progenitores e dos educandos (Bardagi \& Teixeira, 2015; Christofides, Hoy, Milla \& Stengos, 2015; Teixeira \& Bardagi, 2016; Teixeira \& Costa, 2017).

Em síntese, numa visão de modelos holísticos de natureza sociocognitiva, este trabalho visa identificar 
fatores explicativos da autoeficácia geral dos estudantes do ensino superior, que podem ser inspiradores para conceber e implementar intervenções. As hipóteses de investigação decorrem da literatura, de que as crenças de autoeficácia (1) são determinadas por experiências, aprendizagem vicariante, persuasão e estados emocionais, (2) as crenças de capacidade são mais elevadas nos homens do que nas mulheres, e (3) as crenças de capacidade são mais elevadas nos jovens adultos mais velhos do que nos mais novos.

\section{Método}

\section{Participantes}

A amostra é constituída por 480 estudantes do $2^{\circ}$ ano do ensino superior, das áreas da psicologia $(39,7 \%)$, saúde (terapia da fala, terapia ocupacional, enfermagem, fisioterapia) (26\%), desporto (gestão e ciências do desporto) (14\%), científica-investigativa (bioquímica, ciências farmacêuticas) $(4,9 \%)$ e tecnologias (engenharia eletrónica e telecomunicações, engenharia informática e engenharia mecânica) $(15,4 \%)$, em Escolas de Lisboa $(44,4 \%)$, Leiria $(31,6 \%)$ e Algarve (24\%). As mulheres representam 64\% da amostra, variando a idade entre 19 e 34 anos (M 21,06 DP 2,72), e cerca de 60\% têm idades entre 19 e 20 anos. Nos participantes que indicaram as médias de entrada no ensino superior $(n=444)$, as classificações variam entre 10 e $19(M=14,37(D P=2,35)$; cerca de $9 \%$ tem classificações entre 10 e 12 valores, $52 \%$ entre 12,1 e $14,9,33 \%$ entre 15 e 16,9 e $6 \%$ entre 17 e 19 valores. Nos participantes que indicaram as médias do $1^{\circ}$ ano $(n=331)$, as classificações variam entre 8 e 18,2 $(M=13,96$ e $D P=1,60)$.

\section{Instrumentos}

Escala de Autoeficácia Geral (General Self-efficacy Scale)

A escala foi construída por R. Schwarzer e M. Jerusalém (1995), tratando-se de uma escala de autoavaliação das crenças de autoeficácia geral para lidar com situações do quotidiano (e.g., consigo resolver sempre os problemas difíceis, se me esforçar). A escala tem dez itens, formulados no sentido positivo, com resposta Likert de quatro pontos. A investigação tem mostrado coeficientes alfa de Cronbach de 0,76 (Scholz, Dona, Sud \& Scharzer, 2002), 0,84 (Teixeira \& Costa, 2017) e 0,86 (Almeida, 2017). A estrutura unidimensional da medida também tem sido confirmada, em amostras do ensino superior (e.g., Almeida, 2017).
Questionário de Ajustamento e Adaptação ao Ensino Superior

Este questionário foi baseado em instrumentos anteriores sobre transição e adaptação no ensino superior (e.g., Almeida, Soares \& Ferreira, 2002; Soares, Almeida \& Ferreira, 2006). A escala contém 15 itens, nas subescalas: perceção de congruência pessoal (e.g., o curso corresponde às minhas competências /capacidades) (4 itens), perspetiva de empregabilidade-reconhecimento social (e.g., o curso tem boas oportunidades de emprego) (3 itens), social-relacionamento (e.g. relação com os colega (3 itens) e envolvimento no curso (e.g., aquisição de conhecimentos académicos) (4 itens). Estas subescalas correspondem a quatro fatores ortogonais derivados de procedimentos em componentes principais, com rotação varimax (Teixeira \& Costa, 2017). A resposta é dada numa escala de Likert de cinco pontos. Num primeiro estudo com este questionário (Teixeira \& Costa, 2017), os coeficientes alfa de Cronbach variam entre 0,58 e 0,74 . O questionário inclui informações sobre dados sociodemográficos (e.g., sexo, idade) e dados sobre curso, escola e média acesso ao ensino superior.

\section{Escala de Satisfação na Carreira (Career Satisfaction Scale)}

Esta escala foi construída por Greenhaus, Parasuraman e Wormley (1990), com o propósito de avaliar a visão pessoal sobre a progressão da própria carreira de vida (e.g., estou satisfeito(a) com os progressos que fiz para atingir os meus objetivos materiais). É uma escala de autorrelato, unidimensional, com cinco itens e resposta de Likert de cinco pontos. Em diferentes amostras, tem sido confirmada a validade e precisão da medida, nomeadamente a natureza unifatorial e a consistência interna, com coeficientes alfa de Cronbach à volta de 0,85 (Hofmans, Dries \& Pepermans, 2008; Teixeira \& Costa, 2017).

\section{Escala de Desenvolvimento e Bem-estar (Flourishing Scale)}

É um instrumento de autorrelato, que representa aspetos do funcionamento positivo em relações interpessoais, sentimentos de competência, significado e propósito na vida. Trata-se de uma escala unidimensional, com o propósito de avaliar o construto global de bemestar (Diener \& Ryan, 2009). É constituída por 8 itens e a resposta é dada numa escala de Likert de 7 pontos. Em diferentes amostras, tem sido confirmada a validade e 
precisão da medida, nomeadamente a natureza unifatorial e a consistência interna, com coeficientes alfa superiores a 0,87 e de teste-reteste 0,71 (Almeida \& Teixeira, 2018; Diener et al., 2009; Pinto \& Teixeira, 2018; Teixeira \& Costa, 2017).

Inventário de Personalidade (Big five inventory - BFI-10)

O Big Five Inventory (BFI - 10) corresponde a uma das formas reduzidas do Inventário Big Five de McCrae e Costa (1996). Este instrumento contém 10 itens divididos pelos cinco traços principais de personalidade: neuroticismo, extroversão, amabilidade, conscienciosidade e abertura à experiência. A resposta é dada numa escala de Likert de cinco pontos. De acordo com Rammstedt e John (2007), os dois itens de cada uma das escalas foram selecionados pelas boas propriedades psicométricas, e as escalas Extroversão, Conscienciosidade e Neuroticismo revelam bons índices de consistência interna $(0,99-0,82)$ e de estabilidade temporal $(0,83-0,74)$. Em amostras de estudantes universitários ingleses, alemães e portugueses foi identificada uma solução fatorial com cinco fatores, que correspondem às cinco dimensões de personalidade anteriormente enunciados (Costa, 2018; Rammstedt \& John, 2007). Contudo, pelo número reduzido de itens, o instrumento revela fragilidades psicométricas ao nível da consistência interna, situando-se os coeficientes alfa entre 0,36 (Amabilidade) e 0,63 (Extroversão) (Teixeira \& Costa, 2017), e na versão original situam-se entre 0,52 (Amabilidade) e 0,63 (Extroversão) (Rammstedt \& John, 2007).

\section{Escala de Exigência e Responsividade Parental}

Este instrumento foi elaborado por Costa, Teixeira e Gomes (2000), com base nos trabalhos de Maccoby e Martin (1983), com intuito de avaliar padrões educacionais de responsividade e exigência, percecionados pelos adolescentes e jovens adultos. A escala contém vinte e quatro itens para a mãe e outros vinte e quatro para o pai, distribuídos em duas subescalas, que reportam à exigência e responsividade, atribuídas à mãe e ao pai. A resposta é dada numa escala de Likert de cinco pontos. Os conteúdos da escala de exigência são dirigidos para controlo, rigidez, distanciamento, regras e limites. A escala de responsividade inclui conteúdos relativos a empatia, proximidade, apoio emocional e autonomia. Na versão portuguesa, efetuaram-se alterações nos conteúdos de português do Brasil, para português de Portugal (e.g., "me cobra quando eu faço algo errado" para "responsabiliza-me quando faço algo de errado"). Este instrumento evidencia boas qualidades psicométricas, nomeadamente coeficientes alfa de Cronbach de 0,78 nas escalas de exigência e 0,93 nas escalas de responsividade (Teixeira, Bardagi \& Gomes, 2004), sendo superiores a $0,80 \mathrm{em}$ adolescentes portugueses (Bardagi \& Teixeira, 2015). Os dados das versões do Brasil e Portugal mostram convergência na consistência interna e estrutura bifatorial, sendo evidências favoráveis à validade intercultural da medida (Teixeira, Bardagi \& Teixeira, 2016).

\section{Procedimentos}

\section{Éticos}

A investigação foi aprovada pela Comissão de Deontologia da Faculdade de Psicologia da Universidade de Lisboa, em março de 2015. Nas escolas onde foram recolhidos os dados, os diretores das escolas e docentes foram informados dos objetivos do estudo e procedimentos de recolha de dados. Previamente à aplicação dos instrumentos, os participantes assinaram o consentimento informado com garantia de confidencialidade e anonimato.

\section{Recolha de dados}

A aplicação dos questionários foi coletiva, em sala de aula, em cerca de 20 minutos. Na aplicação, procedeu-se à explicação das instruções, solicitando-se a participação pela pertinência dos objetivos do estudo.

A organização do caderno de questionários incluía por ordem o Questionário de Ajustamento e Adaptação ao Ensino Superior, Escala de Satisfação na Carreira, Big 5 Inventory (BFI - 10), Escala de Autoeficácia Geral e Escalas de Exigência e Responsividade Parental.

\section{Análise de dados}

Nas análises estatísticas, procedeu-se ao estudo das distribuições dos resultados e dos coeficientes alfa de Cronbach para averiguar das propriedades psicométricas dos instrumentos, nomeadamente dos indicadores da sensibilidade às diferenças individuais e precisão dos resultados. Foram usados procedimentos de regressão linear múltipla para analisar relações de causalidade e identificar variáveis explicativas da autoeficácia geral. Em sequência, realizaram-se estatísticas inferenciais para esclarecer o modelo de regressão e analisar o efeito do gênero e idade nos resultados. As análises usaram o package estatístico IBM SPSS Statistics versão 24, considerando-se para todas as análises uma probabilidade mínima de erro $(\alpha)$ de 0,05 . 


\section{Resultados}

\section{Precisão, distribuição e correlação dos resultados.}

A Tabela 1 apresenta os coeficientes alfa e as estatísticas descritivas para os seis instrumentos.

No Questionário de Ajustamento e Adaptação ao Ensino Superior, os coeficientes alfa de Cronbach variam entre 0,63 e 0,76, salientando-se coeficientes inferiores a 0,70 , no limite aceitável por alguns autores (Maroco \& Garcia-Marques, 2006). As amplitudes e os desviospadrão mostram variabilidade dos resultados (Tabela 1).
Na Escala Satisfação na Carreira, o coeficiente alfa de Cronbach situa-se em 0,89 , sendo muito favorável à consistência interna da medida. Os indicadores de variabilidade indiciam também sensibilidade da escala às diferenças individuais (Tabela 1).

Na Escala Autoeficácia Geral, o coeficiente alfa situa-se em 0,87 , sendo superior ao da versão original de 0,76 (Sholz et al., 2012). Os indicadores de variabilidade sugerem sensibilidade da medida às diferenças individuais (Tabela 1). Em dois estudos com amostras portuguesas do ensino superior, os coeficientes alfa situam-se em 0,86 (Almeida, 2017) e 0,84 (Teixeira \& Costa, 2017).

Tabela 1

Amplitude, média, desvios-padrão e Alfas de Cronbach

\begin{tabular}{|c|c|c|c|c|}
\hline Escalas & Amplitude & Média & $\mathrm{DP}$ & Alfa \\
\hline \multicolumn{5}{|l|}{ Ajustamento e Adaptação ao Ensino Superior } \\
\hline Empregabilidade e Reconhecimento Social & $4-15$ & 0.72 & 0.72 & 0.76 \\
\hline Congruência & $8-20$ & 0.63 & 0.63 & 0.72 \\
\hline Académico & $8-25$ & 0.66 & 0.66 & 0.63 \\
\hline Relacional-social & $6-15$ & 0.76 & 0.76 & 0.66 \\
\hline Satisfação na carreira & $5-25$ & 18.15 & 3.77 & 0.89 \\
\hline Autoeficácia Geral & $11-40$ & 29.05 & 4.66 & 0.87 \\
\hline \multicolumn{5}{|l|}{ Estilos Parentais } \\
\hline Exigência materna & $13-60$ & 39.01 & 8.61 & 0.84 \\
\hline Responsividade materna & $14-62$ & 50.28 & 9.58 & 0.94 \\
\hline Exigência paterna & $12-59$ & 35.47 & 10.19 & 0.88 \\
\hline Responsividade paterna & $12-60$ & 46.52 & 12.34 & 0.96 \\
\hline \multicolumn{5}{|l|}{ Big Five Inventory (BFI - 10) } \\
\hline Extroversão & $2-10$ & 6.07 & 1.88 & 0.63 \\
\hline Amabilidade & $3-10$ & 7.35 & 1.15 & 0.36 \\
\hline Conscienciosidade & $2-10$ & 6.94 & 1.65 & 0.40 \\
\hline Neuroticismo & 2.10 & 5.89 & 1.92 & 0.54 \\
\hline Abertura à Experiência & $3-10$ & 7.13 & 1.67 & 0.42 \\
\hline Bem-estar & $18-56$ & 43.44 & 6.57 & 0.88 \\
\hline
\end{tabular}

$\mathrm{Na}$ Escala de Perceção Exigência e Responsividade Parental, os alfas variam entre 0,84 e 0,96 . Os índices de variabilidade revelam sensibilidade da medida às diferenças individuais (Tabela 1). Em estudos anteriores, os coeficientes alfa situam-se entre 0,82 e 0,95 (Bardagi \& Teixeira, 2015).

No Questionário Big Five Inventory (BFI - 10), os coeficientes alfa variam entre 0,36 (Amabilidade) e 0,63 (Extroversão). Na versão original da escala, os coeficientes de Amabilidade e Extroversão situam-se respetivamente em 0,52 e 0,63 (Rammstedt \& John, 2007), e em estudantes do ensino superior variam entre 0,48 (Abertura à Experiência) e 0,68 (Extroversão) (Teixeira \& Costa, 2017).
A Escala de Bem-estar apresenta um coeficiente alfa de 0,88 e variabilidade dos resultados. Estes dados são equivalentes aos obtidos em amostras portuguesas (Almeida \& Teixeira, 2018; Pinto \& Teixeira, 2018).

\section{Correlações dos Resultados nos Diferentes Instrumentos}

A Tabela 2 apresenta as intercorrelações dos resultados nos diferentes instrumentos. A amplitude dos coeficientes situa-se entre 0,00 e $|0,65|$. 
Tabela 2

Intercorrelações dos resultados dos diferentes instrumentos

\begin{tabular}{|c|c|c|c|c|c|c|c|c|c|c|c|c|c|c|c|}
\hline Escalas & 1 & 2 & 3 & 4 & 5 & 6 & 7 & 8 & 9 & 10 & 11 & 12 & 13 & 14 & 15 \\
\hline \multicolumn{16}{|l|}{ 1.Satisfação carreira } \\
\hline 2.Extroversao &, $15^{* *}$ & & & & & & & & & & & & & & \\
\hline 3.Amabilidade & ,08 &, $25 * *$ & & & & & & & & & & & & & \\
\hline 4.Conscienciosidade &, $29 * *$ &, $17^{* *}$ &, $11^{*}$ & & & & & & & & & & & & \\
\hline 5.Neuroticismo &,- 07 &,$- 17 * *$ &,- 08 &, 05 & & & & & & & & & & & \\
\hline 6.Abertura & ,09 &, $15^{* *}$ &, $13^{* *}$ &, $09 *$ &,$- 10^{*}$ & & & & & & & & & & \\
\hline 7.Congruência &, $39 * *$ & ,06 & ,09 &, 07 &,- 06 &, 05 & & & & & & & & & \\
\hline 8.Empregabilidade &, $21 * *$ & $10^{*}$ & 01 &,- 07 &,$- 11 *$ & ,04 &, $39 * *$ & & & & & & & & \\
\hline 9.Académico &, $23 * *$ & ,09 &,- 01 &, $12^{* *}$ &,$- 17 * *$ &, 00 &, $24 * *$ & ,09 & & & & & & & \\
\hline 10.Social &, $28 * *$ &, $26 * *$ &, $18 * *$ &, $10^{*}$ &,$- 17 * *$ &,- 02 &, $30 * *$ &, $23 * *$ &, $24 * *$ & & & & & & \\
\hline 11.Autoeficácia &, $31 * *$ &, $18^{* *}$ &,- 00 &, 07 &,$- 37 * *$ &, $23 * *$ &, $18^{* *}$ &, $15^{* *}$ &, $18^{* *}$ &, $16^{* *}$ & & & & & \\
\hline 12.Exigencia mãe & ,08 &, $12 *$ & 07 &, 01 &,- 04 &, $12 *$ &, $12 *$ & ,09 &, 04 & ,06 &,- 02 & & & & \\
\hline 13.Respons. mãe &, $24 * *$ &, $20 * *$ &, $12 *$ &, $18^{* *}$ &,- 04 & $10^{*}$ &, $18 * *$ &, $16^{* *}$ & 07 &, $13 * *$ &, $13 * *$ &, $24 * *$ & & & \\
\hline 14.Exigência pai &, $12 *$ &, $13 * *$ & ,08 &,- 00 &,- 05 &, 05 & ,09 &, $10^{*}$ &, 00 &, $11 *$ &,- 03 &, $65^{* *}$ &, $21 * *$ & & \\
\hline 15.Respons. pai &, $24 * *$ &, $17 * *$ &, $10^{*}$ &, $12 *$ &,$- 13 * *$ &, 02 &, $14 * *$ &, $14 * *$ &, $11 *$ &, $15^{* *}$ &, $14 * *$ &, $25 * *$ &, $56^{* *}$ &, $53 * *$ & \\
\hline 16.Bem-estar &, $51^{* *}$ &, $27 * *$ &, $20 * *$ &, $14^{* *}$ &,$- 28 * *$ &, $11 *$ &, $41 * *$ &, $31 * *$ &, $22 * *$ &, $37 * *$ &, $46^{* *}$ &, 09 &, $41 * *$ &, $13 * *$ &, $29 * *$ \\
\hline
\end{tabular}

$* p<0.05, * * p<0,01$

$\mathrm{Na}$ análise das correlações, considera-se como referência o coeficiente $|0,30|$, que a literatura (e.g., Steele, Andrews, \& Upton, 2012, p. 67) justifica ser uma correlação de força média. Os resultados de Satisfação na Carreira têm associações com Congruência, Autoeficácia e Bem-estar, e os de Neuroticismo correlação negativa com Autoeficácia. Os resultados de Congruência têm ainda associações positivas com Empregabilidade, Social-relacional e Bem-estar. As correlações são superiores a 0,50 entre os resultados das escalas Exigência materna e paterna, Responsividade materna e paterna e Exigência e Responsividade paterna. Os resultados de Bem-estar têm ainda associações positivas com Empregabilidade-reconhecimento social, Social, Autoeficácia e Responsividade materna.

\section{Regressão múltipla linear}

\section{Autoeficácia Geral}

O modelo de regressão múltipla linear inclui Autoeficácia Geral como variável dependente e como variáveis independentes: média de acesso ao ensino superior, satisfação na carreira, gênero, idade, traços de personalidade (Extroversão, Amabilidade, Neuroticismo,
Conscienciosidade, Abertura à Experiência), Estilos Parentais (Exigência e Responsividade da mãe e pai), Vivências Acadêmicas (Congruência, Empregabilidade, Social-Relacional, Envolvimento Acadêmico) e Bemestar. Foram testados os pressupostos do modelo, nomeadamente o da distribuição normal, multicolinearidade, homogeneidade e independência dos erros. Através dos resultados decorrentes da distância de Mahalanobis retiraram-se 2 outliers, com potencial impacto na estimação da reta de regressão; o valor de Durbin-Watson $=2,02$ assegura independência dos resíduos. O modelo de regressão é significativo $\left(R^{2}=0,38\right.$; $F(18,344)=18,34, p<0,001)$, seguiu o método "enter"; $38 \%$ da variabilidade da Autoeficácia Geral é explicado pelas variáveis independentes no modelo. Os valores VIF variam entre 1,08 e 2,81, indicando uma correlação moderada entre as variáveis.

Os coeficientes Beta estandardizados (Tabela 3) indicam como fatores explicativos da Autoeficácia Geral, por ordem: Bem-estar $(p<0,001)$, Neuroticismo (negativo) $(p<0,001)$, Gênero $(p<0,001)$, Satisfação na carreira $(p<0,001)$, Abertura à experiência $(p<0,001)$, Exigência paterna (negativo) $(p<0,05)$, Média de acesso ao ensino superior $(p<0,05)$, Idade $(p<0,05)$ e Amabilidade (negativo) $(p<0,05)$. 
Tabela 3

Coeficientes

\begin{tabular}{lcccccc}
\hline \multicolumn{1}{c}{ V. Independentes significativas } & Beta & Beta padronizados & $t$ & $p$ & Tolerância & VIF \\
\hline (Constante) & 14,98 & & 2,97 &, 00 & & \\
Bem-estar &, 20 &, 29 & 4,79 &, 00 &, 47 & 2,14 \\
Neuroticismo &,- 53 &,- 22 & $-4,81$ &, 00 &, 84 & 1,19 \\
Gênero & $-1,96$ &,- 20 & $-4,16$ &, 00 &, 74 & 1,35 \\
Satisfação carreira &, 24 &, 18 & 3,27 &, 00 &, 55 & 1,81 \\
Abertura à experiência &, 42 &, 14 & 3,33 &, 00 &, 93 & 1,08 \\
Exigência paterna &,- 08 &,- 17 & $-2,47$ &, 01 &, 45 & 2,22 \\
Média acesso ensino superior &, 348 &, 11 & 2,41 &, 02 &, 76 & 1,32 \\
Idade &, 18 &, 10 & 2,11 &, 04 &, 73 & 1,37 \\
Amabilidade &,- 30 &,- 09 & $-2,09$ &, 04 &, 83 & 1,21 \\
\hline
\end{tabular}

\section{Estudo das diferenças entre grupos}

\section{Gênero}

Procedeu-se à análise da diferença dos resultados médios pelo efeito do gênero, pelo teste $t$ de Student, sendo os grupos constituídos por 169 rapazes e 307 meninas. O teste de Levene indica para a maioria das escalas homogeneidade das variâncias, sendo exceção Satisfação na carreira $(p=0,01)$, Empregabilidade-reconhecimento social $(p=0,01)$, Responsividade Paterna $(p=0,01)$, Média de acesso $(p=0,02)$. As diferenças são significativas e favoráveis ao grupo feminino em Média de acesso $(p<0,001)$, Amabilidade $(p<0,001)$, Conscienciosidade $(p<0,001)$, Neuroticismo $(p<0,001)$ e Exigência Materna $(p<0,01)$, sendo favoráveis aos rapazes em Empregabilidade $(p<0,05)$, Autoeficácia Geral $(p<0,001)$ e Bem-estar $(p<0,001)$. De acordo com a classificação de Cohen (1988), a dimensão do efeito é média em Média de acesso, Conscienciosidade, Neuroticismo, Autoeficácia Geral e Bem-estar, sendo pequena em Amabilidade, Exigência Materna e Empregabilidade.

\section{Idade}

Procedeu-se à análise da diferença dos resultados médios pela idade, pelo teste $t$ de Student, sendo os grupos constituídos por 284 participantes com idades entre 19 e 20 anos e 191 entre 21 e 34 anos. O teste de Levene indica homogeneidade das variâncias dos dois grupos na maioria das escalas, sendo exceção Satisfação na carreira $(p=0,02)$, Social $(p=0,01)$ e Exigência materna $(p=0,02)$. Observaram-se diferenças significativas e favoráveis aos participantes mais novos em Satisfação na carreira $(p<0,05)$, Exigência materna $(p<0,001)$ e paterna $(p<0,01)$. As diferenças são significativas e favoráveis ao grupo dos mais velhos em Conscienciosidade $(p<0,05)$ e Autoeficácia geral $(p<0,05)$. A dimensão do efeito é pequena em Satisfação na carreira, Exigência materna e paterna, Autoeficácia geral e Conscienciosidade.

\section{Discussão}

Os resultados são discutidos no âmbito da literatura sociocognitiva (e.g., Bandura, 1977, 1997; Lent \& Brown, 2013; Pajares, 2002), considerando o significado de autoeficácia e das respetivas fontes na relação triádica do funcionamento psicológico (Bandura, 1977, 1997). Salientam-se limitações na amostra e dos índices de precisão do Inventário de Personalidade Big Five Inventory- BFI - 10, que sugerem fragilidade psicométrica da medida. Isto deve-se, em grande parte, ao fato de cada subescala incluir apenas 2 itens (Rammstedt \& John, 2007), e consequentemente resultar pouca variabilidade nos resultados. Em todos os outros instrumentos, os indicadores são favoráveis à precisão das medidas, sendo superiores a 0,80 nas Escala de Bem-Estar, Satisfação na Carreira, Autoeficácia e Perceção de Estilos Parentais. No Questionário de Adaptação e Ajustamento ao Ensino Superior, os coeficientes variam entre 0,76 e 0,66 , sendo ligeiramente superiores aos observados num estudo anterior (Teixeira \& Costa, 2017).

Na premissa de que as crenças de autoeficácia se fundamentam em fontes como experiências, aprendizagem vicariante, persuasão e sentimentos (Bandura, 1977; Usher \& Pajares, 2009), a H1 é parcialmente confirmada pelo modelo de regressão múltipla linear, sendo a Autoeficácia Geral explicada pelas variáveis: Bem-estar, Neuroticismo (negativo), Satisfação na carreira, Abertura à Experiência, Média de acesso ao ensino superior, Exigência paterna (negativo), Amabilidade (negativo), Gênero e Idade. Estes dados podem ser agrupados pelas fontes propostas por 
Bandura (1977, 1997). Assim, a fonte "experiência de mestria" surge representada na Média de acesso ao ensino superior, que em Portugal é fator crítico para os jovens adultos. A entrada para o ensino superior frequentemente representa uma confirmação de capacidade para os estudantes, face a si próprios, à família e aos pares. Estes dados são corroborados pelas evidências (e.g., Dickinson, Abrams \& Tokar, 2017) do peso do desempenho académico nos níveis de autoeficácia, apesar da medida de autoeficácia usada avaliar crenças gerais perante a vida e não capacidades circunscritas aos contextos educacionais e de carreira. Estes resultados confirmam, uma vez mais, o desempenho escolar com grande impacto pessoal, tal como proposto por Bandura (1977), Bandura, Barbaranelli, Caprara e Pastorelli (2001), Bussey e Bandura (1999) e Pajares (2002). Realça-se a possivel contaminação de variáveis de natureza pedagógica, cultural e social nas avaliações, sendo o processo avaliativo objeto de reflexão, para educadores e estudantes.

A fonte "experiências de mestria" pode congregar também o traço Abertura à Experiência, que caracteriza a curiosidade acerca do mundo e a propensão para novas experiências, tão recorrentes no âmbito do ensino superior (Saricaoğlu \& Arslan, 2013). A relação entre Abertura à Experiência e crenças de Autoeficácia é confirmada pelos dados que demonstram esta predisposição pessoal como facilitadora de comportamentos adaptativos, que envolvem desafios, criatividade e resolução de problemas (Lent \& Brown, 2013; Saricaoğlu \& Arslan, 2013). Este resultado pode ser inspirador para as instituições lançarem projetos e atividades dirigidas a estimular e desenvolver estas caraterísticas dos estudantes, em âmbito das atividades curriculares e extracurriculares.

A fonte "estados físicos e emocionais" surge representada nas variáveis Bem-estar, Neuroticismo (negativo) e Satisfação na carreira. Este estudo mostra a relação dos sentimentos de bem-estar, satisfação e a estabilidade emocional (relação inversa de Neuroticismo) com Autoeficácia. A relação negativa do Neuroticismo com Autoeficácia é corroborada na literatura sobre os efeitos nefastos da instabilidade emocional na conceção pessoal de autoeficácia (Lent \& Brown, 2013; Saricaoğlu \& Arslan, 2013). A pesquisa tem confirmado o papel das emoções positivas na motivação e envolvimento nos próprios contextos (Compton, 2005), e o papel atribuído ao optimismo nos domínios intrapessoais e interpessoais (Forgeard \& Seligman, 2012).

Nas categorias das fontes "persuasão e aprendizagem vicariante" incluem-se as variáveis Exigência paterna e Amabilidade (relações negativas), já que são geradores de interação, persuasão e modelagem. Estes dados são corroborados pelo papel da família na construção das crenças de autoeficácia, como agente de modelagem, expetativas e persuasão. As crenças dos educadores sobre as capacidades dos seus educandos influenciam a percepção de autoeficácia dos educandos e a própria concretização dos objetivos académicos e vocacionais (Bandura, 1997; Bandura et al., 2001; Gregory \& Huang, 2013; Teixeira \& Ferreira, no prelo; Tyler \& Boelter, 2008). Por um lado, a pesquisa tem demonstrado associação entre ambientes controladores e fraca motivação intrínseca (Moneta, 2014) e, por outro lado, há evidências da relação entre a atitude de responsividade parental e a confiança dos estudantes para enfrentarem a vida e a carreira (Lent, 2004; Teixeira $\&$ Bardagi, 2016). No mesmo sentido, tem-se confirmado a relação entre autonomia dada pelos pais e o sentimento de competência e bem-estar em contexto académico (e.g., Pesch, Larsin \& Surapaneni, 2016).

A relação negativa entre Autoeficácia e Amabilidade, enquanto predisposição para comportamentos pautados por generosidade, lealdade, cooperação, honestidade e cordialidade e simpatia pode estar influenciada por componentes de dependência relativamente aos outros. Estes dados podem incluir efeitos de gênero e fatores sociais e culturais relacionados com objetivos e projetos de vida (Tate, Fouad, Marks, Young, Guzman, \& Williams, 2015). Salienta-se que apesar da importância do mundo social e emocional no agenciamento pessoal da gestão dos recursos pessoais, ambientais e da própria vida, os estudantes universitários precisam de se sentirem livres de constrangimentos para assumirem com liberdade as suas próprias decisões (Pesch, Larsin \& Surapaneni, 2016).

Este conjunto de fatores que influencia o sentimento de autoeficácia é interpretado no âmbito da relação entre fontes de autoeficácia (Usher \& Pajares, 2009), sendo esta relação inscrita em fatores acadêmicos, sociais e culturais dos próprios contextos (Ahn et al., 2016; Chen \& Usher, 2013; Flores, Navarro, \& Rasheed, 2017; Fouad \& Santana, 2017).

A H2 é confirmada pelos dados da estatística inferencial, que mostram resultados médios superiores dos rapazes em Autoeficácia, e ainda em Bem-estar e percepção de Empregabilidade. Estes dados são corroborados pelos estudos anteriores que confirmam a tendência de os rapazes serem mais autoconfiantes do que as meninas (Almeida, 2017; De Caroli \& Sagone, 2014; Lopes \& Teixeira, 2012; Tong \& Wang, 2017). De destacar a superioridade das médias das meninas nos Resultados escolares, Amabilidade, Conscienciosidade, Neuroticismo e Exigência materna. As diferenças entre as médias favoráveis ao grupo das meninas em Amabilidade são corroboradas pela tendência de a orientação motivacional 
das mulheres ser mais expressiva na relação com as pessoas (Moneta, 2014). A superioridade das médias em Conscienciosidade e Resultados escolares no sexo feminino é corroborada pelos menores índices de abandono escolar, comparativamente aos rapazes, em qualquer um dos ciclos de escolaridade em Portugal (Batista, 2015; Eurydice/Cedefop, 2015), e pela responsabilidade e empenho das meninas no percurso académico (Clinciu, 2013; Gadzella \& Carvalho, 2006). Relativamente ao Neuroticismo, as diferenças de género são corroboradas pela tendência de os elementos do sexo feminino serem mais vulneráveis à ansiedade, nomeadamente em situação de avaliação (Almeida, 2017) e ao estresse no desempenho académico (Clinciu, 2013; Gadzella \& Carvalho, 2006). Contudo, em outras investigações não se registraram diferenças entre sexos em Autoeficácia (Almeida, 2017; Olle, Fouad, Roche, Daskalova, \& Brown, 2017; Siddiqui, 2015) e Bem-estar (Almeida, 2017). A superioridade das médias das meninas em Exigência materna é corroborada na literatura, que explica os estereótipos de género (Baumrind, 1966; Berdagi \& Teixeira, 2015; Costa, Teixeira, \& Gomes, 2000; Teixeira \& Bardagi, 2016). Estes dados sugerem a importância do ambiente familiar na manutenção dos estereótipos de gênero, que subsistem ao longo de gerações.

Os resultados relativos à relação entre gênero, autoeficácia, empregabilidade, resultados escolares, bem-estar e fatores de personalidade podem indiciar a forma desigual como homens e mulheres gerem os seus projetos nos diferentes papéis de vida (Roche, Daskalova \& Brown, 2017).

A H3 é também confirmada pelos dados da estatística inferencial, sendo a média da escala Autoeficácia Geral superior dos alunos mais velhos. Este dado sugere efeitos da aprendizagem nas competências associadas à maturidade psicológica e à adaptação (Almeida, 2017; Gadzella \& Carvalho, 2013; Olle \& Fouad, 2015), e tende a confirmar a possibilidade de educabilidade destas dimensões. Contudo, no estudo de De Caroli e Sagone (2014) não foram encontradas diferenças dos níveis de Autoeficácia Geral pela idade.

\section{Conclusão}

O conjunto dos dados remete para a conceção sociocognitiva, que analisa o conceito de autoeficácia numa ótica autorregulatória e interacionista do funcionamento psicológico. O conjunto dos dados encontra confirmação na literatura (e.g., Bandura, 1977; Usher \& Pajares, 2008), que coloca a tónica na aprendizagem direta e indireta na formação das crenças de autoeficácia, sendo neste estudo confirmada a influência dos fatores dos domínios académico, emocional, social e vocacional, que atuam de modo relacionado, no sistema do autoconceito individual. A informação proporcionada pelas fontes de autoeficácia pode ser usada como elemento orientador das intervenções, no sentido de potenciar a resolução de dificuldades. No caso, por exemplo, do Neuroticismo, podem prever-se intervenções dirigidas à promoção de estratégias de regulação emocional, que promovam estabilidade emocional e bem-estar.

Estes dados também chamam a atenção para a necessidade de organizar estratégias pedagógicas, nas atividades curriculares e extracurriculares, que possam concorrer para a estabilidade emocional e o sucesso dos estudantes. Sublinha-se o papel das redes de suporte ao longo de toda a escolaridade, em que os Serviços de Psicologia e Orientação podem desempenhar um papel de relevância para o estudante, para a família e para a própria comunidade educativa, especialmente os professores.

Este estudo realça ainda o papel dos fatores sociais em traços de personalidade e suporte ambiental da família como promotores de autoconfiança. Os resultados chamam também à atenção sobre a continuidade da influência dos padrões educacionais da família na vida do jovem adulto do ensino superior e da necessidade de intervir com a família, em qualquer fase da vida, de forma a desenvolver padrões de parentalidade responsiva, que promovam competências e atitudes confiantes para enfrentar o mundo académico e social. A presença de suportes sociais e emocionais pode fortalecer a autoeficácia e atuar em termos motivacionais no desempenho. Em futuras pesquisas será pertinente introduzir o domínio dos pares, como suporte de autoeficácia individual.

Por último, salienta-se ainda o impato do gênero no sentimento de autoeficácia e também no bem-estar, o que leva à reflexão sobre as questões das aprendizagens e autoconceito e mesmo sobre o tipo de escolhas e papéis que configuram os projetos dos homens e das mulheres.

Todos estes fatores que explicam a autoeficácia são incentivadores à criação e programação dos serviços de apoio ao estudante por parte das instituições do ensino superior e devem ser considerados no planeamento das intervenções educacionais. Nos objetivos da intervenção salientam-se propósitos de autoconhecimento reflexivo, de promoção de agenciamento pessoal para estabelecer e alcançar objetivos e projetos, de capacidade pessoal para solucionar obstáculos, que possam ser impeditivos do processo adaptativo. Nesta perspetiva, as próprias instituições educativas têm a responsabilidade de proporcionar condições e estruturas de suporte social, que facilitem o florescimento e desenvolvimento no âmbito do ensino superior. 


\section{Limitações do estudo}

A investigação apresenta limitações em temos de método, nomeadamente pela natureza transversal, que restringe o aprofundamento dos mecanismos sociocognitivos envolvidos na relação do sentimento de autoeficácia com o desempenho e sucesso acadêmicos. De realçar, uma vez mais ainda, as limitações das caraterísticas metrológicas do Inventário de Personalidade BFI - 10, especialmente quando nesta investigação os fatores de personalidade se tornam tão significativos para explicar autoeficácia. Outra limitação prende-se com o facto de a amostra ser composta maioritariamente por estudantes de Psicologia e elevada percentagem de mulheres. Em estudos futuros será necessário procurar amostras com maior diversidade em cursos, percursos acadêmicos e outras variáveis relevantes para aprofundar a relação entre autoeficácia, desempenho e bem-estar.

\section{Referências}

Ahn, H. S., Usher, E. L., Butz, A., \& Song, M. (2016). Cultural differences in the understanding of modelling and feedback as sources of self-efficacy information. British Journal of Educational Psychology, 86(1), 112-136. doi: 10.1111/bjep.12093

Almeida, B. R. (2017). Gapar ou não Gapar, importa explorar e apoiar! A influência de atividades exploratórias e de suporte na transição e adaptação ao ensino superior (tese de Mestrado). Faculdade de Psicologia: Lisboa.

Almeida, B. M., \& Teixeira, M. O. (2018). Bem-estar e adaptabilidade de carreira na adaptação ao ensino superior. Revista Brasileira de Orientação Profissional, 19(1), 19-30. DOI: http://dx.doi.org/1026707/1984-7270/2019v19n1p19

Almeida, L. S., Soares, A., \& Ferreira, A. (2002). Questionário de Vivências Académicas (QVA-r): Avaliação do Ajustamento dos Estudantes Universitários. Avaliação Psicológica, 2, 81-93.

Almeida, L. S., Araújo A. M., \& Martins, C. (2016). Transição e adaptação dos estudantes do $1^{0}$ ano: variáveis intervenientes e medidas de atuação. In L. S. Almeida \& R. V. Castro (Orgs.), Ser estudante no ensino superior: o caso dos estudantes do $1^{\circ}$ ano (pp. 146-164). Braga: Universidade do Minho, Centro de Investigação em Educação.

Alvan, S., Belgrave, F., \& Zea, M. (1996). Stress, social support, and college adjustment among latino students. Cultural diversity and mental health, 2(3), 193-203.

Bandura, A. (1977). Self-efficacy: Toward a Unifying Theory of Behavioral Change. Psychological Review, 84(2), 191-215.

Bandura, A. (1995). Self-Efficacy in changing societies. Cambridge, UK: Cambridge University Press.

Bandura, A. (1997). Self-efficacy: The exercise of control. New York: Freeman.

Bandura, A., Barbaranelli, C., Caprara, G. V., \& Pastorelli, C. (2001). Self-efficacy beliefs as shapers of children's aspirations and career trajectories. Child Development Journal, 72(1), 187-206.

Bardagi, M., \& Hutz, C. (2008). Apoio Parental percebido no contexto da escolha inicial e da evasão do curso universitário. Revista Brasileira de Orientação Profissional, 9(2), 31-44.

Bardagi, M. P. \& Teixeira, M. O. (2015, julho). Psychometrical properties of Portuguese and Brazilian versions of the Parental Demandingness and Responsiveness Scale: An intercultural study. Comunicação apresentada no 13th European Conference on Psychological Assessment. Universidade de Zurique: Zurique.

Batista, J. O. (2015). Indicadores de transferência e de abandono no ensino superior português. Seminário sucesso académico 2015. Recuperado de http://www.dgeec.mec.pt/np4/\%7B \$clientServletPath\%7D/?newsId=499\&fileN ame $=2 \_$Apresentacao_DGEEC.pdf

Baumrind, D. (1966). Effects of Authoritative Parental Control on Child Behavior. Child Development, 37(4), 887-907.

Betz, N. E., \& Hackett, G. (1981). The relationship of career-related self-efficacy expectations to perceived career options in college women and men. Journal of Counseling Psychology, 28, 399-410.

Bussey, K. \& Bandura, A. (1999). Social cognitive theory of gender development and differentiation. Psychological Review, 106(4), 67-713.

Chen, J. A., \& Usher, E. L. (2013). Profiles of the sources of science self-efficacy. Learning and Individual Differences, 24, 11-21.

Christofides, L. N., Hoy, M., Milla, J., \& Stengos, T. (2015). Grades, Aspirations, and Postsecondary Education Outcomes. Canadian Journal of Higher Education, 45(1), 48-82. Recuperado em 27 de setembro de 2017, de http://journals.sfu. ca/cjhe/index.php/cjhe/article/viewFile/184203/pdf_1

Clinciu, A. (2013). Adaptation and stress for the first-year university students. Procedia-Social and Behavioral Sciences, 78, 718-722. doi: 10.1016/j.sbspro.2013.04.382. 
Compton, W. C. (2005). An Introduction to Positive Psychology. Virginia: Thomson/Wadsworth.

Costa, C. J. M. (2018). Bem-estar e satisfação na carreira em estudantes do ensino superior. Dissertação de doutoramento não publicada. Faculdade de Psicologia da Universidade de Lisboa: Lisboa.

Costa, F. T., Teixeira, M. A. P., \& Gomes, W. B. (2000). Responsividade e exigência: Duas escalas para avaliar estilos parentais. Psicologia: Reflexão e Crítica, 13, 465-473. Recuperado em 27 de setembro de 2017, de http://www. scielo.br/pdf/prc/v13n3/v13n3a14.pdf

De Caroli, M. E., \& Sagone, E. (2014). Generalized self-efficacy and well-being in adolescents with high vs. low scholastic self-efficacy. Procedia. Social and Behavioral Sciences, 141, 867-874. Retirado de http://creativecommons.org/ licenses/by-nc-nd/3.0/.

Dickinson,J, Abrams, M. D. \& \& Tokar, D. M. (2017). An Examination of the Applicability of Social Cognitive Career Theory for African American College Students. Journal of Career Assessment, 25(1) 75-92. DOI: $10.1177 / 1069072716658648$.

Diener, E., \& Ryan K. (2009). Subjective well-being: a general overview. Journal of Psychology, 39(4), 391-406.

Direção-Geral de Estatísticas da Educação e Ciência (2015). Indicadores de transferência e de abandono no ensino superior português. Retirado de http://www.dgeec.mec.pt/np4/292/\%7B \$clientServletPath\%7D/?newsId=516\&file Name $=$ Indicadores_transferencia_abandono__Ens.pdf

Eurydice Brief. (2015). Tackling early leaving from education and training in Europe. doi:10.2797/259399

Ferreira, F., \& Fernandes, P. (2015). Fatores que influenciam o abandono no ensino superior e iniciativas para a sua prevenção: O olhar de estudantes. Educação, Sociedade e Culturas, 45, 117-197.

Flores, L. Y., Navarro, R. L., \& Rasheed, S. (2017). The state of SCCT research in relation to social class: future directions. Journal of Career Assessment, 25(1), 6-23. doi: 10.1177/1069072716658649.

Forgeard, M. J. C., \& Seligman, M. E. P. (2012). Seeing the glass half full: A review of the causes and consequences of optimism. Pratiques Psychologiques, 18(2), 107-120.

Fouad, N., \& Santana, M. C. (2017). SCCT and underrepresented populations in STEM Fields: moving the needle. Journal of Career Assessment, 27, 24-39.

Greenhaus, J. H., Parasuraman, S., \& Wormley, W. M. (1990). Effects of race on organizational experiences, job performance evaluations, and career outcomes. Academy of Management Journal, 33(1), 64-86.

Gadzella, B. M., \& Carvalho, C. (2006). Stress differences among university female students. American Journal of Psychological Research, 2(1). Retirado de https://www.mcneese.edu/f/c/79a44c7a/AJPR\%2006-05\%20 Gadzella.pdf

Gregory, A., \& Huang, F. (2013). It takes a village: The effects of 10th grade college-going expectations of students, parents, and teachers four years later. American Journal of Community Psychology, 52(1-2), 41-55.

Hill, N. E., \& Wang, M-T. (2015). From middle school to college: Developing aspirations, promoting engagement, and indirect pathways from parenting to post high school 18 enrollment. Developmental Psychology, 51(2), 224-235. doi:10.1037/a003836710.1037/a0038367.

Hofmans, J., Dries, N., \& Pepermans, R. (2008). The Career Satisfaction Scale: Response bias among men and women. Journal of Vocational Behaviour, 73, 397-403.

Høigaard, R., Kovac, V. B., Øverby, N. C., \& Haugen, T. (2015). Academic Self-Efficacy Mediates the Effects of School Psychological Climate on Academic Achievement. School Psychology Quarterly, 30(1), 64-74.

Hutz, C. S., \& Bardagi, M. P. (2006). Indecisão profissional, ansiedade e depressão na adolescência: a influência dos estilos parentais. Psico-USF, 11(1), 65-73.

Lent, R. W., \& Brown, S. D. (2006). Integrating person and situation perspectives on work satisfaction: A social-cognitive view. Journal of Vocational Behavior, 69, 236-247. DOI: 10.1016/j.jvb.2006.02.006.

Lent, R. W., \& Brown, S. D. (2013). Social cognitive model of career self-management: Toward a unifying view of adaptive career behavior across the life span. Journal of Counseling Psychology, 60(4), 557-568. http://dx.doi. org $/ 10.1037 / \mathrm{a} 0033446$

Lopes, A. R. \& Teixeira, M. O. (2012). Projetos de carreira, autoeficácia e sucesso escolar em ambiente multicultural. Revista Brasileira de Orientação Profissional, 13(1), 7-14. Recuperado em 27 de setembro de 2017, de http://pepsic. bvsalud.org/pdf/rbop/v13n1/03.pdf

Luszczynska, A., Gutiérrez-Doña, B., \& Schwarzer, R. (2004). General self-efficacy in various domains of human functioning: Evidence from five countries. International Journal of Psychology, 40(2), 80-89. 
Luszczynska A., Scholz, U., \& Schwarzer, R. (2005). The General Self-Efficacy Scale: Multicultural Validation Studies. The Journal of Psychology, 139(5), 439-457. doi: 10.3200/JRLP.139.5.439-457

Maroco, J. \& Garcia-Marques, T. (2006). Qual a fiabilidade do alfa de Cronbach? Questões antigas e soluções modernas? Laboratório de Psicologia, 4(1), 65-90.

Maccoby, E. E., \& Martin, J. A. (1983). Socialization in the context of the family: Parent- child interaction. New York: Wiley.

McCrae, R. R., \& Costa, P. T., Jr. (1996). Toward a new generation of personality theories: Theoretical contexts for the five-factor model. In J. S. Wiggins (Ed.), The five-factor model of personality: Theoretical perspectives, (p. 51-87). New York: Guilford.

Moneta, G. B. (2014). Posite psychology: A critical introduction. NY: Palgrave Macmillan.

Olle, C. D., \& Fouad, N. A. (2015). Parental support, critical consciousness, and agency in career decision making for urban students. Journal of Career Assessment, 23(4), 533-544.

Pajares, F. (2002). Gender and Perceived Self-Efficacy in Self-Regulated Learning. Theory Into Practice, 41(2), 116-125.

Pesch, K. M, Larson, L. M \& Surapaneni, S. (2016). Parental Autonomy Support and Career Well-Being: Mediating Effects of Perceived Academic Competence and Volitional Autonomy. Journal of Career Assessment, 24(3) 497-512. DOI: $10.1177 / 1069072715599392$.

Pinto, A. B. \& Teixeira, M. O. (2018). Bem-estar subjetivo em Alunos das Escolas Profissionais. In M. C. Taveira, A. D. Silva, C. Marques \& M. Leal (Cord. e Org.), Carreira e aconselhamento: Educação, Mobilidade e Emprego (pp.150162). Braga: APDC Edições.

Rammstedt, B. \& Jonh, O. (2007). Mesuring personality in one minute or less: A 10-item short version of the Big Five Inventory in English and German. Journal of Research in Personality, 41, 203-212. DOI: 10.1016/j. jrp.2006.02.001.

Roche, M. K, Daskalova, P \& Brown, S.D. (2017). Anticipated Multiple Role Management in Emerging Adults: A Test of the Social Cognitive Career Self-Management Model. Journal of Career Assessment, 25(1), 121-134. DOI: $10.1177 / 1069072716658654$.

Saricaoğlu, H \& Arslan, C. (2013). An Investigation into Psychological Well-being Levels of Higher Education Students with Respect to Personality. Educational Sciences: Theory \& Practice, 13(4). doi: 10.12738/estp.2013.4.1740

Scholz, U., Dona, G., Sud, S., \& Scharzer, R. (2002). Is general self-efficacy a universal construct? Psychometric findings from 25 countries. European Journal of Psychology Assessment, 18(3), 242-251.

Schwarzer, R. \& Jerusalem, M. (1995). Generalized Self-Efficacy scale. In J. Weinman, S. Wright, \& M. Johnston, Measures in health psychology: A user's portfolio. Causal and control beliefs (pp. 35-37). Windsor, UK: Nfer-Nelson.

Siddiqui, S. (2015). Impact of self-efficacy on psychological well-being among undergraduate students. The International Journal of Indian Psychology, 2(3), 2349-3429.

Silva, R. S., \& Nascimento, I. (2014). Ensino Superior e Desenvolvimento de Competências Transversais em Futuros Economistas e Gestores. Revista Brasileira de Orientação Vocacional, 15(2), 225-236.

Soares, A., Almeida, L. S., \& Ferreira, J. (2006). Questionário de Vivencias Academicas: Versão integral (QVA) e versão reduzida (QVA-r). In M. Goncalves, L. Almeida, \& M. Simões (Coords.), Avaliação psicológica: Instrumentos validados para a população portuguesa (2 $2^{\mathrm{a}}$ ed. atualizada, Vol. 1, pp. 101-120). Coimbra: Quarteto.

Steele, C., Andrews, H., \& Upton. D. (2012). Statistics in psychology. Harlow: Pearson Education.

Tate, K. K. A., Fouad, N. A, Marks, L, R., Young, G., Guzman, E \& Williams, E. G. (2015). Underrepresented First-Generation, Low-Income College Students' Pursuit of a Graduate Education: Investigating the Influence of Self-Efficacy, Coping Efficacy, and Family Influence. Journal of Career Assessment, 23(3) 427-441. DOI: $10.1177 / 1069072714547498$

Teixeira, M. A. P., Bardagi, M.P. \& Gomes, W. B. (2004). Refinamento de um instrumento para avaliar responsividade e exigência parental percebidas na adolescência. Avaliação Psicológica, 3(1), 1-12. Universidade Federal do Rio Grande do Sul. Rio Grande do Sul.

Teixeira, M. O. (2008). A abordagem sócio-cognitiva no aconselhamento vocacional: Uma reflexão sobre a evolução dos conceitos e da prática da orientação. Revista Brasileira de Orientação Profissional, 9(2), 9-16.

Teixeira, M. O. \& Bardagi, M. (2016). Relationship between parenting styles and career development variables in Portuguese students. In Tamara Ramiro-Sánchez \& $\mathrm{M}^{\mathrm{a}}$ Teresa Ramiro (Coord.). Avances enciencias de la educación 
$y$ deldesarrollo, 2015, (1080-1086). Asociación Española de Psicología Conductual (AEPC). Recuperado: http:// congresoeducacion.es/edu_web4/capitulos_2015.pdf.

Teixeira, M.O., Bardagi, M. P. \& Teixeira, M. (2016, setembro). Estudo intercultural dos Estilos de Parentalidade. Comunicação apresentada no Simpósio Contextos de desenvolvimento da carreira e de vida. Comunicação no $3^{\circ}$ Congresso da Ordem dos Psicólogos Portugueses. Porto.

Teixeira, M. O. \& Costa, C. J. (2017). Carreira e bem-estar subjetivo no ensino superior. Revista Brasileira de Orientação Profissional, 18(1), 19-29.

Teixeira, M. O., \& Ferreira, I. A. (no prelo). Fatores familiares e escolares nas aspirações, na autoeficácia e no sucesso académico: um estudo exploratório com estudantes de $9^{\circ}$ ano. Revista Portuguesa de Psicologia.

Tong, K. K., \& Wang, Y. Y. (2017). Validation of the flourishing scale and scale of positive and negative experience in a Chinese community sample. PLoS ONE 12(8), 1-10. doi: https://doi. org/10.1371/journal.pone.0181616

Tyler, K. M., \& Boelter, C. M. (2008). Linking Black Middle School Students' Perceptions of Teachers' Expectations to Academic Engagement and Efficacy. Negro Educational Review, 59(1-2), 27-44.

Usher, E. L., \& Pajares, F. (2009). Sources of Self-Efficacy in Mathematics: A Validation Study. Contemporary Educational Psychology, 34(1), 89-101.

Recebido 16/02/2018

$1^{a}$ Reformulação 02/08/2018

$2^{a}$ Reformulação 31/08/2018

$3^{a}$ Reformulação 06/12/2018

Aceite Final 08/01/2019

Sobre as autoras

Maria Odília Teixeira é Professora Auxiliar da Faculdade de Psicologia da Universidade de Lisboa. Especialista no domínio do aconselhamento de carreira, com diversas publicações neste campo.

Cátia João Costa é Doutorada em Psicologia da Educação. Psicóloga Clínica com funções na Santa Casa da Misericórdia de Porto de Mós. 